IdeAs

Idées d'Amériques

16 | 2020

Les marges créatrices : intellectuel.le.s afrodescendant.e.s et indigènes auX Amériques, XIX-XXe siècle

\title{
Inscrire l'égalité des sexes dans la Constitution états-unienne : Equal Rights Amendment, promesses et déboires d'une campagne interminée
}

Claire Delahaye

\section{OpenEdition}

Journals

Édition électronique

URL : http://journals.openedition.org/ideas/9617

DOI : $10.4000 /$ ideas.9617

ISSN : 1950-5701

Éditeur

Institut des Amériques

Référence électronique

Claire Delahaye, «Inscrire l'égalité des sexes dans la Constitution états-unienne : Equal Rights

Amendment, promesses et déboires d'une campagne interminée ", IdeAs [En ligne], 16 | 2020, mis en ligne le 01 octobre 2020, consulté le 18 octobre 2020. URL : http://journals.openedition.org/ideas/ 9617 ; DOI : https://doi.org/10.4000/ideas.9617

Ce document a été généré automatiquement le 18 octobre 2020

\section{c)}

IdeAs - Idées d'Amériques est mis à disposition selon les termes de la licence Creative Commons Attribution - Pas d'Utilisation Commerciale - Pas de Modification 4.0 International. 


\title{
Inscrire l'égalité des sexes dans la Constitution états-unienne : Equal Rights Amendment, promesses et déboires d'une campagne interminée
}

\author{
Claire Delahaye
}

1 De nombreux médias états-uniens l'ont souligné au début de l'année 2020 : ne serait-ce pas un symbole fort et une victoire extraordinaire de voir l'amendement pour l'égalité des droits, Equal Rights Amendment (ERA), ajouté à la Constitution états-unienne l'année où le centenaire du $19^{\mathrm{e}}$ amendement était célébré ?

2 Ce qui a suscité cet engouement fut la ratification de l'amendement par l'État de Virginie le 15 janvier, amenant le nombre d'États ayant voté l'ERA à 38, ce qui correspond aux trois quarts requis pour l'entériner, après que le Nevada et l'Illinois l'ont ratifié en 2017 et 2018 respectivement. L'hostilité d'acteurs et d'actrices de la sphère publique états-unienne s'est également exprimée, à l'image du leader des Républicains au Congrès, le sénateur Mitch McConnell, déclarant son opposition à l'ERA le 5 février, lors d'une conférence de presse.

3 Ces événements ont donc relancé le débat sur un projet né dans les années 1920, aux enjeux complexes articulant questions idéologiques, politiques, économiques et culturelles, aux ramifications constitutionnelles, philosophiques et militantes. Pourquoi cet amendement est-il l'objet de controverses et de crispations si tenaces ? Cet article propose de revenir sur son histoire et d'explorer les débats qu'il a pu susciter, afin d'examiner comment la question de l'égalité a pu s'inscrire dans des enjeux politiques et institutionnels. 


\section{Du droit de vote à l'ERA, appropriation et usages politiques du passé}

4 L'amendement a été débattu plusieurs fois au Congrès depuis sa première proposition en décembre 1923 par deux Républicains, le sénateur Charles Curtis et le représentant Daniel Anthony, le neveu de Susan B. Anthony (1820-1906), célèbre militante pour les droits des femmes. Lors de ces nombreux débats, il n'est pas rare que l'ERA soit inscrit dans un héritage historique, celui du droit de vote, plusieurs membres du Congrès évoquant par ailleurs l'histoire longue des combats législatifs des femmes pour leurs droits. Cette représentation historique symptomatique d'une vision particulière, marquée par le progrès, témoigne d'une minimisation des expériences opprimées et minoritaires (le droit de vote est ainsi loin d'être acquis pour les Américain.e.s racialisé.e.s même après l'adoption du $19^{\mathrm{e}}$ amendement en 1920), révélant des phénomènes d'appropriations et des usages politiques du passé, auxquels les militantes qui ont rédigé l'amendement ont eu elles-mêmes recours. En effet, le texte de l'ERA a été pensé par la militante suffragiste Alice Paul dès 1921, et il fut présenté officiellement en 1923, lors de cérémonies commémoratives élaborées par son organisation le National Woman's Party (NWP) en l'honneur du $75^{\mathrm{e}}$ anniversaire de la première convention pour les droits des femmes de Seneca Falls. Ce groupe se revendiquait comme l'unique héritier légitime de tout un mouvement complexe, d'autant qu'il ne s'était pas dissout et avait conservé le même nom après l'adoption du droit de vote des femmes. Grâce à l'ERA, le NWP souhaitait lutter contre toutes les lois qui discriminaient les Américaines, concernant par exemple leur droit à accéder à la fonction de juré, à conserver leur nom et leur domicile si elles se mariaient, ou à contrôler leurs biens. Ce projet fut officiellement discuté en février 1921 à la première convention du NWP après la ratification du $19^{e}$ amendement, et il fut dès son origine source de controverse.

\section{Un amendement clivant}

5 En effet, les discussions autour de l'amendement lors de la convention de 1921 furent particulièrement houleuses et révélèrent d'importants clivages idéologiques et politiques. Notons tout d'abord que les déléguées noires, qui sollicitèrent l'appui des militantes pour lutter contre les discriminations dont elles étaient victimes et qui alertèrent sur le fait qu'elles ne pouvaient toujours pas voter dans le Sud, ne furent pas écoutées. Ironiquement, l'heure était à des débats autour de la possibilité de garantir l'égalité grâce à un amendement constitutionnel.

6 Lors des discussions, les féministes s'affrontèrent notamment autour de la question des lois protégeant les femmes au travail, leur garantissant par exemple des conditions décentes ou des horaires adaptés. Beaucoup craignaient que ces lois, souvent le fruit d'âpres luttes, fussent remises en cause si un amendement était adopté : était-il possible d'affirmer l'égalité des droits, tout en conservant des lois fondées sur la différence des sexes? Au début des années 1920, Alice Paul estimait que tel était bien le cas, et elle espérait que l'égalité permettrait aux hommes de bénéficier des mêmes protections que les femmes, mais elle devint convaincue que ces lois étaient un handicap à la réussite économique de celles-ci et les traitaient comme des êtres inférieurs. Elle envisageait l'égalité comme une égalité des chances. D'autres 
réformatrices considéraient au contraire qu'il fallait prendre en compte la situation spécifique des femmes (maternité, travail domestique) dans la lutte pour l'égalité.

7 S'opposaient aussi plusieurs visions du travail, liées à des enjeux de classe et de race : même si les discriminations envers les femmes et leur exploitation étaient incontestables, certaines envisageaient le travail salarié comme une libération et la forme d'indépendance à privilégier, même si les femmes ne gagnaient pas assez pour vivre. Plusieurs militantes en faveur de l'ERA estimaient que les lois protégeant les femmes jouaient contre elles sur le marché du travail et les maintenaient dans des positions subalternes. Cette position révélait un statut privilégié, de femmes pouvant ignorer d'autres formes de discrimination que celles liées au sexe, parce qu'elles leur étaient invisibles. C'est ainsi que la vision d'un féminisme individualiste défendue par le NWP devint le signe d'un féminisme élitiste, réservé à des femmes blanches éduquées et de classes supérieures, même si des travailleuses dans le domaine de l'imprimerie, de la restauration ou des transports purent partager leur vision en déplorant les limitations légales qui leur étaient imposées, concernant par exemple leurs horaires de travail.

Cette approche divisa profondément les mouvements féministes: dès 1922, de puissantes organisations nationales telles que les League of Women Voters, Woman's Trade Union League, General Federation of Women's Clubs ou National Consumers League affirmèrent publiquement leur opposition à l'ERA. Dans le contexte politique des années 1920, marqué par une doctrine du laissez-faire, l'ERA pouvait conduire à un désengagement de l'État quant à la régulation du travail. Face aux ravages du capitalisme industriel et aux rudes batailles menées contre l'exploitation des femmes, un tel projet semblait donc inacceptable pour nombre de militantes. La décision de la Cour Suprême en 1923, déclarant des lois assurant un salaire minimum pour les femmes dans certains États comme anticonstitutionnelles, sembla leur donner raison. De surcroît, la position du NWP conduisit l'organisation à forger des alliances problématiques : en 1931, menant campagne en Géorgie contre les lois interdisant le travail de nuit, il se trouva, en défendant la liberté contractuelle, du côté des conservateurs hostiles aux syndicats et à toute réforme raciale. Puisque le NWP s'était approprié la lutte pour l'égalité par le biais d'un amendement à la Constitution, l'ERA ne rencontra pas l'appui espéré et l'organisation fut perçue comme nuisible. La stratégie du NWP révélait une vision étriquée de la justice sociale, fondée uniquement sur le genre, qui ne prenait pas en compte l'expérience des femmes pauvres ou racialisées, et les militantes engagées dans des syndicats ou organisations de gauche, qui pensaient qu'il était du devoir de l'État de corriger les inégalités, ne pouvaient se revendiquer d'un tel féminisme perçu comme réactionnaire.

En outre, la nécessité d'un amendement pour assurer l'égalité pouvait également faire débat. En effet, comme l'avait souligné Florence Kelley, à la tête de la puissante National Consumers League dans les années 1920 et 1930 et farouche opposante à l'ERA, l'interprétation qui avait été faite par les tribunaux du $14^{\mathrm{e}}$ amendement garantissant les droits des citoyens et citoyennes avait totalement dévoyé l'objectif du texte, qui avait fini par assurer une protection aux corporations et non aux anciens et anciennes esclaves. Selon elle, chercher à codifier l'égalité sociale et politique dans la Constitution pouvait avoir des effets imprévisibles et indésirables (Storrs L., $2000: 48$ ). 


\section{L'ERA au Congrès entre 1940 et 1970}

10 Si l'ERA a pu diviser les féministes, l'amendement fut néanmoins introduit à chaque session au Congrès entre 1923 et 1972. Le texte fut examiné en commission parlementaire 32 fois entre 1923 et 1946, et il fut voté et approuvé pour la première fois au Sénat en 1946. La même année, 10 personnalités, dont l'ancienne première dame Eleanor Roosevelt et Frances Perkins, l'ancienne ministre du Travail du gouvernement de Franklin Delano Roosevelt, publièrent un communiqué dénonçant l'ERA comme étant susceptible d'invalider toute loi auparavant adoptée pour protéger les femmes au travail. En 1950 et 1953, un texte modifié fut approuvé par le Sénat, incluant l'avenant Hayden (d'après le nom d'un sénateur de l'Arizona), qui stipulait que l'amendement ne pourrait pas permettre de retirer aux femmes les droits ou les protections qui leur étaient garantis par la loi. Mais la Chambre des représentants ignora l'ERA entre 1948 et 1970, notamment parce que le représentant de New York Emmanuel Celler, proche des organisations syndicales, s'opposait à ce que la Commission des lois qu'il présidait examinât l'amendement (Neale T., 2019: 9-11). Soulignons par ailleurs que le Parti républicain inclut l'ERA dans son programme à partir de 1940 et jusqu'en 1980. Les Démocrates firent de même, de 1944 jusqu'à aujourd'hui.

\section{Remobilisation dans les années 1960 et 1970}

11 L'Equal Pay Act de 1963 et le Civil Rights Act de 1964 offrirent un terrain législatif propice à un renouveau de l'ERA, mais ce furent également les mobilisations féministes de l'époque qui redonnèrent un second souffle au combat. L'association National Organization for Women fit de l'ERA sa priorité et l'opposition des organisations syndicales s'estompa, à l'image de United Auto Workers, le plus grand syndicat national, qui apporta son soutien à l'amendement en avril 1970. L'ERA fut adopté en 1972 par le Congrès, avec l'appui du président Nixon, et envoyé aux cinquante États pour être ratifié dans un délai de 7 ans. En 1972, 22 États ratifièrent l'amendement, ils furent 8 en 1973, 3 en 1974, et 1 en 1975 puis en 1977. Alors que la date butoir approchait, le Congrès permit une extension de 38 mois pour la ratification, jusqu'en 1982, alors que cinq États avaient adopté des mesures pour annuler leur ratification (mais la constitutionnalité de ces mesures fait encore débat). Ce ralentissement dans la ratification de l'amendement est attribué au mouvement contre l'ERA, incarné par des lobbys et l'opposition de législateurs, mais rendu visible par une coalition regroupant plusieurs organisations conservatrices, notamment Eagle Forum de Phyllis Schlafly.

\section{L'opposition s'organise}

Il semblerait que l'opinion publique états-unienne a toujours approuvé l'ERA, puisque les résultats des sondages ne descendirent jamais en dessous de $54 \%$ d'avis favorables, mais la campagne «STOP ERA » de Phyllis Schlafly a mis à mal l'idée selon laquelle il y avait un consensus en faveur de l'amendement chez les femmes. Son succès révèle un enjeu de représentation démocratique, à deux niveaux, puisqu'il a d'une part érodé le soutien législatif à l'ERA, et a d'autre part interrogé sa légitimité à incarner l'opinion de la majorité des Américaines. 
13 L'historienne Susan Marshall (1991) note des similitudes pertinentes entre le mouvement contre le $19^{\mathrm{e}}$ amendement et celui contre l'ERA, notamment leur nature profondément réactionnaire, même s'ils sont pétris de contradiction, puisque les militantes participèrent à la sphère politique pour soutenir une vision traditionnelle de la division sexuée du travail et de la famille, tout en minorant leur organisation structurelle pour amplifier le sentiment d'une opposition populaire. L'hostilité à l'ERA était plus importante chez les blanches, mariées, plus âgées, et moins éduquées que chez les Africaines Américaines, les femmes célibataires ou divorcées, plus jeunes, et diplômées du supérieur. Mais il ne faut pas oublier que contrairement à la représentation d'un « crêpage de chignon entre femmes » comme le déplorent Eleanor Smeal et Gloria Steinem (2020) à propos de la série Mrs. America, l'opposition à l'ERA est surtout incarnée par des hommes, qu'ils soient à la tête de corporations ou élus dans les assemblées représentatives dans les États ou au niveau fédéral (Suk J., 2020).

Les arguments utilisés par « STOP ERA » renversèrent souvent l'idée d'émancipation, en faisant de l'amendement une nouvelle forme d'asservissement : arguer par exemple en pleine guerre du Vietnam que les femmes seraient tenues de servir sous les drapeaux put être particulièrement fructueux. Les opposants et opposantes à l'ERA aujourd'hui reprennent nombre de ces raisonnements, en alléguant que les femmes pourront être envoyées en première ligne au combat, que les employeurs ne seront plus tenus de fournir des aménagements particuliers pour les femmes enceintes, que l'avortement serait définitivement libéralisé, les prisons, les toilettes et les foyers d'accueil seraient mixtes. Selon ces personnes, l'ERA signifierait donc un recul, illustrant qu'un progrès social en vue d'une plus grande égalité peut être vécu comme une menace ou une perte.

\section{Conclusion}

Dans le contexte actuel, la ratification de l'ERA semble loin d'être acquise, et le débat qu'il suscite encore illustre de profonds clivages politiques, idéologiques et culturels, mais révèle également des enjeux constitutionnels importants, comme la légitimité même d'un amendement à la Constitution, le processus de son adoption et de sa ratification.

16 Le 13 février dernier, la Chambre des représentants a voté un projet de loi, qui supprime la date butoir pour ratifier l'ERA, avec l'appui de tous les Démocrates et cinq Républicains, mais le Sénat, à majorité républicaine, risque de s'y opposer. Lors des débats à la Chambre, les Républicain.e.s ont particulièrement insisté sur la question de l'avortement. Certain.e.s objectent également que ce n'est pas au Congrès, mais aux tribunaux et éventuellement à la Cour Suprême de décider de la constitutionnalité de la ratification de l'ERA. Plusieurs actions en justice sont déjà en cours, dans le Massachusetts, à Washington D.C. ou dans l'Alabama. La juge à la Cour Suprême Ruth Bader Ginsburg, qui avait soutenu l'ERA dans les années 1970, s'est d'ailleurs exprimée sur la question, soulignant la contradiction qu'il y avait à refuser le recul sur la ratification de certains États, tout en acceptant les nouvelles ratifications (Savage D., 2020). Le gouvernement de Donald Trump a clairement fait part de son hostilité à cet amendement, notamment par le biais d'une note publiée par le ministère de la Justice.

L'opposition à l'ERA, malgré un soutien indéfectible de l'opinion publique, peut servir d'illustration à une crise profonde de la démocratie étatsunienne, du point de vue de 
ses institutions mais aussi de sa représentativité, liée à la surreprésentation politique et idéologique d'une frange conservatrice de la population.

\section{BIBLIOGRAPHIE}

Marshall, Susan E., « Who Speaks for American Women? The Future of Antifeminism », The Annals of the American Academy of Political and Social Science 515, 1991, p. 50-62, www.jstor.org/ stable/1046927, page consultée le 15 avril 2020.

Neale, Thomas H., « The Proposed Equal Rights Amendment: Contemporary Ratification Issues », Congressional Research Service Report $n^{\circ} 42979$, Washington, D.C., Congressional Research Service, 2018, updated December 23, 2019, https://crsreports.congress.gov

Savage, David G., « Ratification of Equal Rights Amendment runs into opposition - from Trump, sure, but Ruth Bader Ginsburg ? » Los Angeles Times, 13 février 2020, https://www.latimes.com/ politics/story/2020-02-13/ratification-of-era-looks-doubtful-ginsburg-skepticism, page consultée le 5 juillet 2020 .

Smeal, Eleanor and Gloria Steinem, «Steinem and Smeal : Why 'Mrs. America' is bad for American women », Los Angeles Times, 30 juillet 2020, https://www.latimes.com/entertainmentarts/tv/story/2020-07-30/steinem-and-smeal-why-mrs-america-is-bad-for-american-women, page consultée le 30 juillet 2020 .

Storrs, Landon R. Y., Civilizing Capitalism : The National Consumers' League, Women's Activism, and Labor Standards in the New Deal Era, Chapel Hill and London, University of North Carolina Press, 2000.

Suk, Julie. C., « Op-Ed : Now, as in the 1970s, it's men, not women, who will defeat the Equal Rights Amendment », Los Angeles Times, 9 août 2020, https://www.latimes.com/opinion/story/ 2020-08-09/kate-blanchett-phyllis-schlafly-equal-rights-amendment-era-mitch-mcconnell, page consultée le 23 août 2020.

\section{AUTEUR}

\section{CLAIRE DELAHAYE}

Université Gustave Eiffel 